\title{
Angiogenesis inhibitors in combinatorial approaches
}

\author{
Patrycja Nowak-Sliwinska $^{1} \cdot$ Arjan W. Griffioen $^{2}$
}

Received: 7 February 2017/Accepted: 12 March 2017/Published online: 5 April 2017

(C) Springer Science+Business Media Dordrecht 2017

It is generally thought that angiogenesis inhibitors, when applied for the treatment of cancer, show their best clinical benefit in well-designed combination approaches. Such combinations can be based on mixtures of different angiogenesis inhibitors, allowing the advantage of improved efficacy by targeting different signaling pathways [1-3]. In addition, it is believed that the risk of druginduced resistance is reduced when inhibition is affected through multiple pathways [4]. Alternatively, the inhibition of blood vessel formation can be combined with other promising treatment regimens. Both strategies are subject of the current special issue of Angiogenesis. This special issue addresses the current and timely efforts and advances in preclinical and clinical studies in combinatorial approaches involving angiogenesis inhibitors. Six dedicated articles show different perspectives of possible alleys of improving the activity of angiogenesis inhibitors via combining them with other treatment strategies. In renal cell cancer the approval of the first angiostatic tyrosine kinase inhibitors induced a renaissance in the treatment of this disease. While this type of cancer used to be practically untreatable, the application of sunitinib showed an immediate response in about half of the patients. Although combination with other targeted drugs was either difficult or did not further improve survival, the combination with

Patrycja Nowak-Sliwinska

patrycja.nowak-sliwinska@unige.ch

$\triangle$ Arjan W. Griffioen

a.griffioen@vumc.nl

1 School of Pharmaceutical Sciences, University of Geneva, 1211 Geneva, Switzerland

2 Department of Medical Oncology, VU University Medical Center, 1007 MB Amsterdam, The Netherlands immunotherapy is currently extremely promising. Kuusk et al. [5] review the current clinical trials on combination therapies of VEGF-targeted therapy with the so-called immune checkpoint inhibitors in metastatic renal cell carcinoma. Another paper by Ramjiawan et al. [6] continues on the importance of immunotherapy in combination with anti-angiogenic agents and addresses the mechanisms of reprogramming the tumor microenvironment in order to enhance tumor autoimmunity and circumvent resistance to angiostatic drugs. A different insight into current angiostatic combination trials in the clinic is provided by Hamming et al. [7], providing an overview of the combination of radiotherapy with angiostatic agents. The authors elegantly discuss the results of recently performed clinical trials. Bani et al. [8] explore the mechanisms of anti-angiogenic tyrosine kinase inhibitors in combination with chemotherapeutics. In a set of new preclinical data they present the fascinating activity of p-glycoprotein and its role in tumor endothelial cell chemoresistance. A promising effort in the development of anticancer drugs is the approach of targeting epigenetics of cancer. Berndsen et al. [9] examine the role of epigenetic drugs as angiostatics, as well as the application of epigenetic drugs in combination with regular angiostatic-targeted agents. The authors present an extensive literature review of various classes of epigenetic compounds, both clinically approved and experimental compounds that are paving the way to effective combination strategies. Last but not least, van Beijnum et al. [10] address the question whether microRNAs are promising candidates to successful anti-angiogenic-based treatment of cancer.

These publications all together present a broad spectrum of combinatory approaches describing opportunities for improved cancer treatment. Although many questions remain to be answered, general interest in combination 
strategies seems to drive the whole field of cancer research. We hope that this special issue facilitates the recognition and understanding of the power of carefully designed combination regimens.

\section{References}

1. Griffioen AW, Weiss A, Berndsen RH, Abdul UK, te Winkel MT, Nowak-Sliwinska P (2014) The emerging quest for the optimal angiostatic combination therapy. Biochem Soc Trans 42(6):1608-1615. doi:10.1042/BST20140193

2. Weiss A, Ding X, van Beijnum JR, Wong I, Wong TJ, Berndsen RH, Dormond O, Dallinga M, Shen L, Schlingemann RO, Pili R, Ho CM, Dyson PJ, van den Bergh H, Griffioen AW, NowakSliwinska P (2015) Rapid optimization of drug combinations for the optimal angiostatic treatment of cancer. Angiogenesis 18(3):233-244. doi:10.1007/s10456-015-9462-9

3. Nowak-Sliwinska P, Weiss A, Ding X, Dyson PJ, van den Bergh $\mathrm{H}$, Griffioen AW, Ho CM (2016) Optimization of drug combinations using Feedback System Control. Nat Protoc 11(2):302-315. doi:10.1038/nprot.2016.017

4. van Beijnum JR, Nowak-Sliwinska P, Huijbers EJ, Thijssen VL, Griffioen AW (2015) The great escape; the hallmarks of resistance to anti-angiogenic therapy. Pharmacol Rev 67(2):441-461
5. Kuusk T, Albiges L, Escudier B, Grivas N, Haanen J, Powles T, Bex A (2017) Antiangiogenic therapy combined with immune checkpoint blockade in renal cancer. Angiogenesis. doi:10.1007/ s10456-017-9550-0

6. Ramjiawan RR, Griffioen AW, Duda DG (2017) Anti-angiogenesis for cancer revisited: Is there a role for combinations with immunotherapy? Angiogenesis. doi:10.1007/s10456-017-9552-y

7. Hamming LC, Slotman BJ, Verheul HMV, Thijssen VJ (2017) The clinical application of angiostatic therapy in combination with radiotherapy: past, present, future. Angiogenesis. doi:10. 1007/s10456-017-9546-9

8. Bani MR, Decio A, Giavazzi R, Ghilardi C (2017) Contribution of tumor endothelial cells to drug resistance: anti-angiogenic tyrosine kinase inhibitors act as p-glycoprotein antagonists. Angiogenesis. doi:10.1007/s10456-017-9549-6

9. Berndsen B, Abdul UK, Weiss A, Zoetemelk M, Te Winkel MT, Dyson PJ, Griffioen AW, Nowak-Sliwinska P (2017) Epigenetic approach for angiostatic therapy; promising combinations for cancer treatment. Angiogenesis. doi:10.1007/s10456-017-9551-Z

10. van Beijnum JR, Giovannetti E, Poel D, Nowak-Sliwinska P, Griffioen AW (2017) miRNAs: microManagers of anti-cancer combination therapies. Angiogenesis. doi:10.1007/s10456-0179545-x 